\title{
Study of Fractal Aperture Distribution and Flow in Fractures
}

\author{
S. Kumar, R. W. Zimmerman, and G.S. Bodvarsson \\ Earh Sciences Division \\ Lawrence Berkeley Laboratory \\ University of California \\ Berkeley, California 94720
}

January 1990

This work was supponed by the U.S. Deperument of Energy under Contract No. DE-AC03-76SF00098, administered by the DOE Nevada OIfice, in cooperation with the U.S. Geological Survey, Denver. 


\title{
STUDY OF FRACTAL APERTURE DISTRIBUTION \\ AND FLOW IN FRACTURES
}

\author{
S. KUMAR, R.W. ZIMMERMAN, and G.S. BODVARSSON \\ Earth Sciences Division \\ Lawrence Berkeley Laboratory \\ University of Califomia \\ Berkeley, CA 94720 \\ (415) 486-7419,-7106,-4789
}

\begin{abstract}
This study examines the roughness profiles and aperture distributions of fractures and faults by using concepts from fractal geometry. Simple models of flow of fluid in rough fractures are also discussed. A deterministic fractal representation of the roughness profile is presented which is shown to have many distinct advantages over other numerical methods, such as information compression, uniqueness and repeatability of surface simulation, retention of statistical information, and self-similarity over many scales. Also the fractal representation enables an isotropic surface and an aperture distribution to be simulated by examining a measured profile. Saturated fluid flow in fractures is then computed using a combined Navier-Stokes and Darcy equation.
\end{abstract}




\section{INTRODUCTION}

Fluid flow in fractures plays an important role in the characterization of the unsaturated zone at Yucca Mountain, the proposed nuclear repository site. Various tests are and will be conducted to investigate the fracture characteristics, and their abilities to transmit fluid under different unsaturated conditions. As a part of this effort a large number of roughness profiles of fracture surfaces are expected to be measured and analyzed. ${ }^{1}$ In this work we investigate the fractal characteristics of fracture surfaces and develop a methodology for estimating the aperture distributions from roughness profiles. A simple model of fluid flow in fractures $:$, isented whi a is illustrated by examining idealized case of fractures with cylindritul asperities a.: the effects of drag by the asperities are evaluated.

Surfaces of fractures and faults exhibit two distinct properties that make them amenable for analysis by the application of the theory of fractal geometry. The first is that roughness profiles of the surface seem to be non-differentiable, though they are continuous. The other is that the profile is self-similar at different scales over a large range of length scales. Previous studies in the literature have established the fractal dimension of various geophysical surfaces but have relied on numerical simulations based on random number generators to simulate ${ }^{2}$ and study ${ }^{3}$ these surfaces. The present analysis focuses on a deterministic representation of the roughness profile and the rough surface in order to reduce the computational effort as well as to derive semi-analytical expressions for various surface characteristics. The deterministic technique enables an isotrcpic surface and an aperture distribution between opposing surfaces to be created by examining one profile.

A fractal representation has the advantage that it preserves the self-similarity exhibited by the surface roughness and retains the statistical information without resorting to large numerical simulations. With the selection of a few constants the deterministic formulation uniquely re-creates the rough profile of the surface. This yields a large information compression. Additionally, the deterministic formulation is independent of any kind of mesh spacing. A final advantage of the deterministic fractal formulation is that the various values of interest (such as contact area) can be evaluated semi-analytically. 
The flow of fluid over fracture surfaces is difficult to analyze because of the complicated flow paths of the fluid between discrete asperities. The asperities are not uniform in height, shapes, nor spacings, and follow a statistical distribution. Upon encountering an obstacle in the form of a solid asperity the fluid has to go around or above it, depending on the path of least resistance. The present study introduces a simple model to analyze fluid flow over rough surfaces. Assuming that the fluid always goes around the asperity along the plane of the mean flow, an effective permeability is defined to account for the viscous and pressure drag by the asperities. The resultant flow is modeled by a combination of Stokes and Darcy flows, thereby reducing the mathematical complexity of the original three-dimensional Navier-Stokes flow.

\section{FRACTAL REPRESENTATION OF ROUGHNESS}

The deterministic formulation selected for the representation of surface roughness profile is the Weierstrass-Mandelbrot cosine fractal function $W(x)$ which is expressed as $^{4-7}$

$$
z(x)=W(x)=A \sum_{n=n_{1}}^{n_{2}} \frac{\cos \left(2 \pi \beta^{n} x\right)}{\beta^{(2-D) n}}, 1<D<2
$$

where $D$ is the fractal dimension of the roughness profile, $\beta$ is a non-integral constant greater than unity, and $A$ is a scaling constant. The above function $W$ is continuous at all points but has no derivative anywhere. The frequencies of $W$ are in geometric progression and, if the point $x=0$ is avoided, the phases of different modes do not coincide. This gives the resultant profile a random appearance. The power spectrum (spectral density) $S(\omega)$ of the above profile representation is proportional to $\omega^{-(5-2 D)}$ and is expressed as

$$
S(\omega)=\frac{A^{2}}{2} \sum_{n=n 1}^{n 2} \frac{\delta\left(\omega-2 \pi \beta^{n}\right)}{\beta^{(4-2 D) n}}
$$




$$
=\frac{A^{2}(2 \pi)^{(4-2 D)}}{2 \ln \beta} \frac{1}{\omega^{(5-2 D)}} .
$$

Here the zero frequency and negative frequencies have been neglected. Experimentally observed profiles of fracture and other rock surfaces show similar straight line behavior on $\log -\log$ plots 8 indicating that the above representation is a valid one.

The statistical parameters such as mean square height of a profile are not expected to be identical to that of a surface since the profile is expected to miss most of the highest peaks and deepest troughs on the surface. Assuming that the autocorrelation functions of the profile and the surface are similar (i.e., the surface is isotropic), a mathematical development ${ }^{3}$ can be followed which yields the relationship between the surface and profile power spectrums:

$$
S_{s}(\omega)=\frac{(5-2 D) B(3-D, 0.5)}{\pi} \frac{S(\omega)}{\omega} .
$$

where $B$ is the Beta function.

In this study the following function which satisfies the above relation, and is based on the Weierstrass-Mandelbrot function, is used to represent rough surfaces:

$$
\frac{z_{s}(x, y)}{F(D)}=A \sum_{n=n !}^{n 2} \frac{\cos \left(\sqrt{2} \pi \beta^{n} x\right) \cos \left(\sqrt{2} \pi \beta^{n} y\right)}{\beta^{(2-D) n}} .
$$

Here $F(D)$ is a scaling function that compensates for the additional cosine term in order to yield profiles that are similar is magnitude to those presented in the previous section. Since the above expression is symmetric about $x=y$, it is a good representation of the surface away from this line. The power spectrum of the above representation is

$$
S_{s}\left(\omega_{x}, \omega_{y}\right)=\frac{A^{2} F^{2}(D)}{4} \sum_{n=n 1}^{n 2} \frac{\delta\left(\omega_{x}-\sqrt{2} \pi \beta^{n}\right) \delta\left(\omega_{y}-\sqrt{2} \pi \beta^{n}\right)}{\beta^{(4-2 D) n}}
$$


Converting the above from a Cartesian to a cylindrical representation yields

$$
\begin{aligned}
S(\omega) & =\frac{A^{2} F^{2}(D)}{4 \pi^{2}} \sum_{n=n 1}^{n 2} \frac{\delta\left(\omega-2 \pi \beta^{n}\right)}{\beta^{(4-2 D) n}} \\
& \approx \frac{A^{2}(2 \pi)^{(4-2 D)}}{2 \ln \beta} \frac{F^{2}(D)}{\pi} \frac{1}{\omega^{(6-2 D)}} .
\end{aligned}
$$

Comparing the above expression with the previous prediction (3) yields the value of $F(D)$. Using the above formulations it can be shown that the ratio of mean square height of the surface to that of the profile is equal to $F^{2}(D) / 2$ which equals unity if $D=2$. This is consistent with the physical intuition since for a fractal dimension of 2 the surface is extremely jagged (e.g. white noise) and the profile does not miss even the highest peaks and the deepest valleys.

Anisotropy is encountered in many geologic surfaces. Brown and Scholz ${ }^{8}$ observed that the fractal dimensions along the two principal directions of anisotropy do not differ significantly for siltstone bedding surfaces altered by frictional wear from glaciation as well as for anisotropic joints in siltstone. The power spectrums of the surface profiles deviate slightly at large wavelengths, with profiles perpendicular to the wear grooves having more power. The anisotropy of such surfaces is evident visually, even though its manifestation in the power spectrum is not significant. If it is assumed that the fractal dimension of the surface remains same in all directions then these weakly anisotropic surfaces are represented by the above formulation by introducing $\beta_{x}$ and $\beta_{y}$ in the cosines and letting $\beta$ in the denominator to be the smaller of $\beta_{x}$ and $\beta_{y}$. The ratio $\beta_{x} / \beta_{y}$ is determined by the degree of anisotropy. If for example $\beta_{y}$ is larger than $\beta_{x}$ the discrete power spectrum is lower for the profiles in the $y$ direction. Various parameters to quantify the anisotropic nature of the surface may then be easily obtained by manipulating the above formulation.

The aperture distribution due to two opposing rough surfaces can now be evaluated as (see Figure 1) 


$$
z_{a}(x, y)=z_{s 1}(x, y)+z_{s 2}(x, y)+d .
$$

where $d$ is the dilatancy of the fracture. Contact areas are defined as those where $z_{a} \leq$ 0 . Since the mean values $\left\langle z_{s 1}\right\rangle$ and $\left\langle z_{s 2}\right\rangle$ are zero, the mean value $\left\langle z_{a}\right\rangle$ is $d$. The above can be simplified by letting the two surfaces to be mirror images with shear displacements $x_{c}$ and $y_{c}$ in the $x$ and $y$ directions, i.e., $z_{s 2}(x, y)=-z_{s 1}\left(x+x_{c}, y+y_{c}\right)$. For such a case the power spectrum is given as

$$
S_{a}\left(\omega_{x}, \omega_{y}\right)=2\left[1-\cos \left(\omega_{x} x_{c}+\omega_{y} y_{c}\right)\right] S_{s}\left(\omega_{x}, \omega_{y}\right)
$$

where negative frequencies as well as the zero frequency term have been neglected.

\section{Numerical Study}

Three parameters have to be selected to numerically study and simulate roughness: $\beta, n_{1}$, and $n_{2}$. The parameter $\beta$ is conveniently selected as 1.5. Parameter $n_{1}$ is selected so that the largest wavelength corresponds approximately to the sample length $L$. If the surface being simulated is a one which has been experimentally measured by a stylus then $n_{2}$ is selected to match the smallest wavelength approximately equal to the stylus tip dimensions. The dimension $D$ of the profile is obtained by analyzing the slope of power spectrum of the experimentally obtained roughness profile. The scaling constant $A$ is evaluated by examining the area under the spectral density curve. A profile simulated by using the Weierstrass-Mandelbrot function is presented in Figure 2. The parameters used in generating this profile are obtained by analyzing the profile of Brown ${ }^{9}$ which yields: $D=1.5, n_{1}=-16, n_{2}=-2, A=0.03234$, and $\beta=1.5$. The value of $x$ is from 2700 to 3700 . Figure 3 presents a surface generated by the present scheme corresponding to the profile presented in Figure 2.

\section{FLOW IN FRACTURES}

In this section we evaluate the effects of asperities on the global permeabilities of fractures. It is assumed that on $x-y$ planes along the direction of the mean flow, the fluid streams around the roughness asperities. The average flow along this two- 
dimensional plane is characterized by an effective permeability $K_{2}$ and porosity $\phi$, where $K_{2}$ is the resultant effect of he viscous and pressure drags exerted by the asperities to the flow of fluid in the plane. Both $K_{2}$ and $\phi$ are functions of $z$, and the porosity $\phi(z)$ is defined as the open area for fluid flow at a transverse location $z$. The porosity is evaluated by employing relations from fractal theory to obtain analytical expressions. ${ }^{6}$ The effective permeability $K_{2}(z)$ has to be computed by solving the two-dimensional Stokes equations in the $x-y$ plane. Since the roughness elements are highly irregular in size the results of Hasimoto ${ }^{10}$ and Sangani and Yao, ${ }^{11}$ who analyzed Stokes flow through random distributions of cylinders, are used.

The time-dependent equations that govern flow within the fracture are obtained by combining the two-dimensional Navier-Stokes equations with the effective permeability concept (cf. Brinkman, ${ }^{12}$ Vafai and Tien ${ }^{13}$ ):

$$
\frac{\rho}{\phi^{2}(z)} \frac{\partial \bar{u}(x, z, t)}{\partial t}=-\frac{d(\varphi+\rho g x)}{d x}-\frac{\mu}{K_{2}(z)} \bar{u}(x, z, t)+\frac{\mu}{\phi^{2}(z)} \frac{\partial^{2} \bar{u}(x, z, t)}{\partial z^{2}}
$$

The velocity $\overline{\boldsymbol{u}}$ is averaged locally over the $x-y$ plane, and hence is a function only of $x$ and $z$. This velocity is a "Darcy velocity" and can be related to the actual average velocity of the fluid particles by $\bar{u}=\phi^{2} u$ (actual). One factor of $\phi$ accounts for the reduced area available for flow, while the second is introduced here to account for "tortuosity", in the sense that the actual travel path of a fluid particle between two points $x=x_{1}$ and $x=x_{2}$ must exceed the distance $\left|x_{2}-x_{1}\right|$. Although the identification of the tortuosity with $1 / 4$ is not exact, this idea has had some success in predicting the electrical conductivity of porous rocks.

\section{Idealized Fracture}

A fracture consisting of smooth parallel walls separated by a distance $h$ containing randomly distributed cylindrical asperities is considered (see Figure 4). The governing can be analytically integrated for steady state, constant $\phi$ and $K_{2}$ yielding

$$
\bar{u}(z)=\frac{-K_{2}}{\mu} \frac{d p}{d x}\left[1-\frac{\cosh \left(\phi 2 / \sqrt{K_{2}}\right)}{\cosh \left[\phi h / 2 \sqrt{K_{2}}\right]}\right] .
$$


The total volumetric flux can be found by averaging the velocity again, this time over the $z$ direction. The equivalent "three-dimensional" fracture permeability $K_{3}$ is found by comparing it with Darcy's law to yield

$$
K_{3}=K_{2}\left[1-\frac{\tanh \left(\phi h / 2 \sqrt{K_{2}}\right)}{\left(\phi h / 2 \sqrt{K_{2}}\right]}\right] .
$$

The above expression reduces to the expected results in the two limiting cases of $\phi \rightarrow 1$ (no obstacles), and $h \rightarrow \infty$ (no side walls). In the former case, note that $K_{2} \rightarrow \infty$ when $\phi \rightarrow 1$ with $h$ held constant, so that $K_{3} \rightarrow h^{2} / 12$ as $\phi \rightarrow 1$, which is the known permeability for flow between parallel flat plates. In the other limit of $h \rightarrow \infty$ (with $\phi$ held fixed), $\tanh (\infty)=l$ in $(10)$, so that $K_{3} \rightarrow K_{2}$ as $h \rightarrow \infty$. In this case the permeability reduces to that of flow across an array of infinitely long, parallel circular cylinders. There is another important limit in which $h l a \rightarrow 0$ for fixed $c,(c=1-\phi)$, in which case (10) reduces to $K_{3}=\phi^{2} h^{2} / 12=h^{2} / 12(1-c)^{2}$. If the concentration of obstacles $c$ is small but finite, the above expression reduces to $(1-2 c) h^{2} / 12$, which is the result found by Walsh ${ }^{14}$ for 2 thin fracture containing a small concentration of randomly distributed circular asperities. If one of the $\boldsymbol{K}_{2}$ expressions from literature is used, such as by :Tasimoto ${ }^{10}$ for small values of $c$

$$
K_{2}=\frac{a^{2}}{8 c}(-\ln c-1.476+2 c+\ldots)
$$

and that of Sangani and $\mathrm{YaO}^{11}$ for higher $c$

$$
K_{2}=\frac{a^{2}}{3.34 c}[1-1.10 \sqrt{c}]^{5 / 2} \text {, }
$$

the three-dimensional permeability $K_{3}$ can be predicted (Figure 5). Here $a$ is root mean square radius of the cylindrical asperities. It is seen that for very thin fractures 
the wall shear dominates and the reduction in permeability is due to tortuosity of the flow that is introduced due to asperities. For larger values of $h$ more asperity surface is exposed to the flow and asperity viscous shear drag begins to be significant. Since most fractures have asperity contact areas less than 30\%, either expression of $K_{2}$ used yields similar results.

\section{CONCLUSIONS}

The deterministic scheme studied in the present work to generate roughness profiles and rough surfaces has tremendous advantages over conventional numerical algorithms for generating and visual rendering of random processes. The major advantages are the extreme information compression, repeatability, saving of computer time and storage, ant extrapolating the information from profiles to generate rough surfaces and aperture distributions. Another feature of the present scheme, not available via simple conventional algorithms, is the ease of generation of anisotropic surfaces. The present method is thus a powerful tool in the study of rough surfaces and the various phenomena associated with them.

A new simple model for analyzing flow over rough surfaces has been developed. To iliustrate the approach an expression has been derived for the permeability of a fracture that consists of two smooth parallel walls and contains cylindrical asperities. The predicted permeability reduces to that of an assemblage of infinitely long parallel cylinders when the aperture is very large, and to the parallel plate permeability when the asperity concentration goes to zero. The results show that viscous drag along the faces of asperities appreciably reduces the permeability of a fracture.

\section{ACKNOWLEDGEMENTS}

This work was carried out under U.S. Department of Energy Contract No. DEAC03-765F00098, administered by the DOE Nevada Office, in cooperation with the U.S. Geological Survey, Denver. The authors thank K. Karasaki and J.S.Y. Wang of LBL for helpful comments and discussions. 


\section{REFERENCES}

1. P. HARROLD, C.C. BARTON, and P. MONTAZER, "Statistical analysis of fracture roughness profiles in volcanic tuffs, Yucca Mountain, Nevada," EOS, 66(46), p. 883 (1985).

2. S.R. BROWN, "Transport of fluid and electric current through a single fracture," Journal of Geophysical Research, 94, p. 9429 (1989).

3. J.S.Y. WANG, T.N. NARASIMHAN, and C.H. SCHOLZ, "Aperture correlation of a fractal fracture," Journal of Geophysical Research, 93, ․ 2216 (1988).

4. M.V. BERRY, and Z.V. LEWIS, "On the Weierstrass-Mandelbrot fractal function," Proceedings of the Royal Society of London, A-370, p. 459 (1980).

5. B.B. MANDELBROT, The Fractal Geometry of Nanure, W.H. Freman, New York (1982).

6. J. FEDER, Fractals, Plenum Press, New York (1998).

7. A. MAJUMDAR, "Fractal surfaces and their applications to surface phenomena," $P h D$ Dissertation, University of Califormia, Berkeley (1989).

8. S.R. BROWN, and C.H. SCHOLZ, "Broad bandwidth study of the topography of natural rock surfaces," Journal of Geophysical Research, 90, p. 12575 (1985).

9. S.R. BROWN, "A note on the description of surtace roughness using fractal dimension," Geophysical Research Letrers, 14, p. 1095 (1987).

10. H. HASIMOTO, "On the periodic fundar ental solutions of Stokes equations and their application to viscous flow past a cubic aray of cylinders," Journal of Fluid Mechanics, 5, p. 317 (1959).

11. A.S. SANGANI, and C. YAO, "Transport processes in random arrays of cylinders. II. Viscous flow," Physics of Fluids, 31, p. 2435 (1988).

12. H.C. BRINKMAN, "A calculation of the viscous force exerted by a flowing fluid on a dense swarm of particles," Applied Science Research, Al, p. 27 (1947).

13. K. VAFAI, and C.L. TIEN, "Boundary and inertial effects on flow and heat transfer in porous media," International Journal of Heat and Mass Transfer, 24, p. 195 (1981).

14. J.B. WALSH, "Effect of pore pressure and confining pressure on fracture permeability," International Journal of Rock Mechanics, 18, p. 429 (1981). 


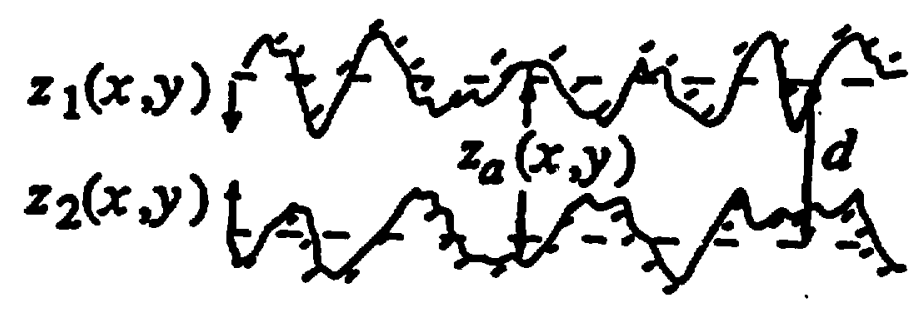

FIG. 1. Schematic depiction of apertures. 


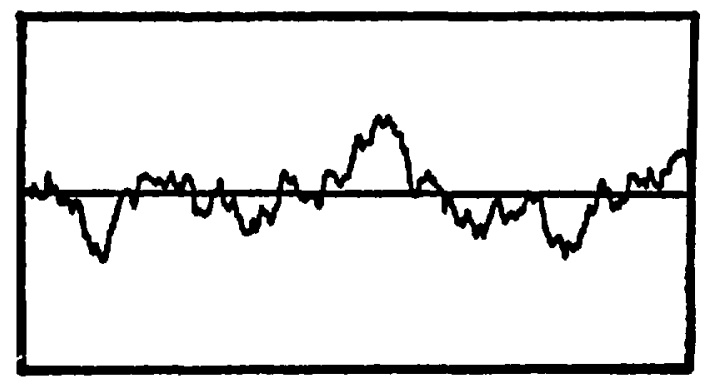

FIG. 2. Fractal profile of dimension $D=1.5$ generated by present scheme. 


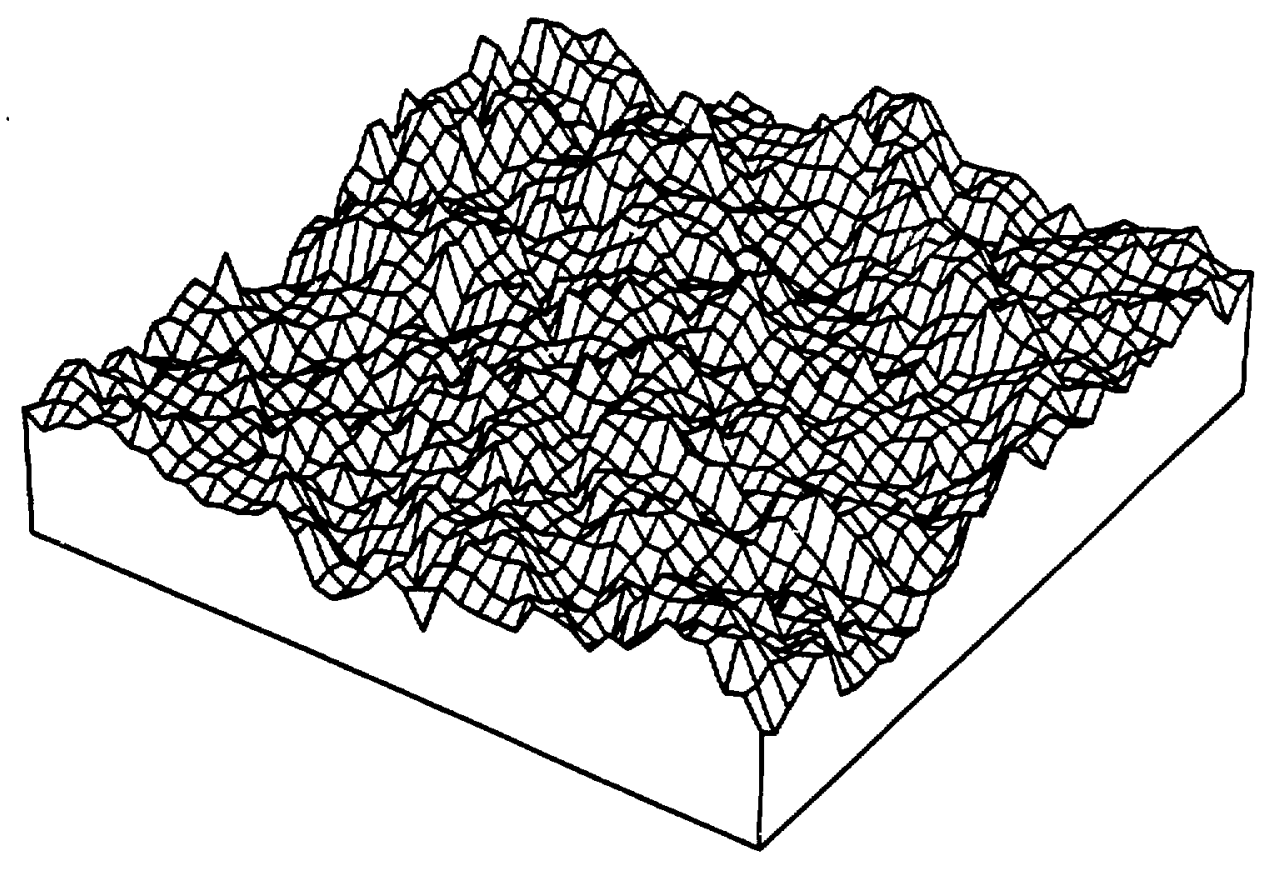

FIG. 3. Surface generated by present scheme. 

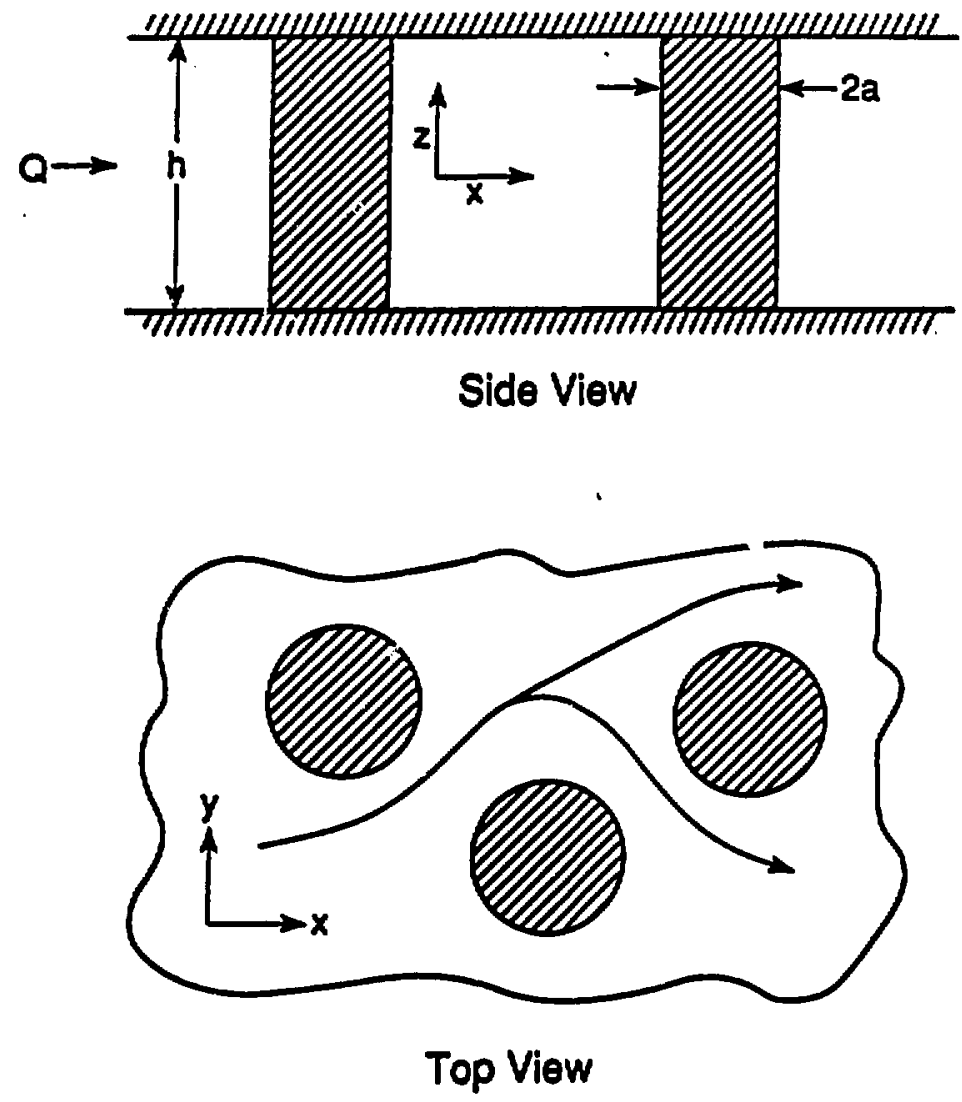

FIG. 4. Schematic of an idealized fracture. 


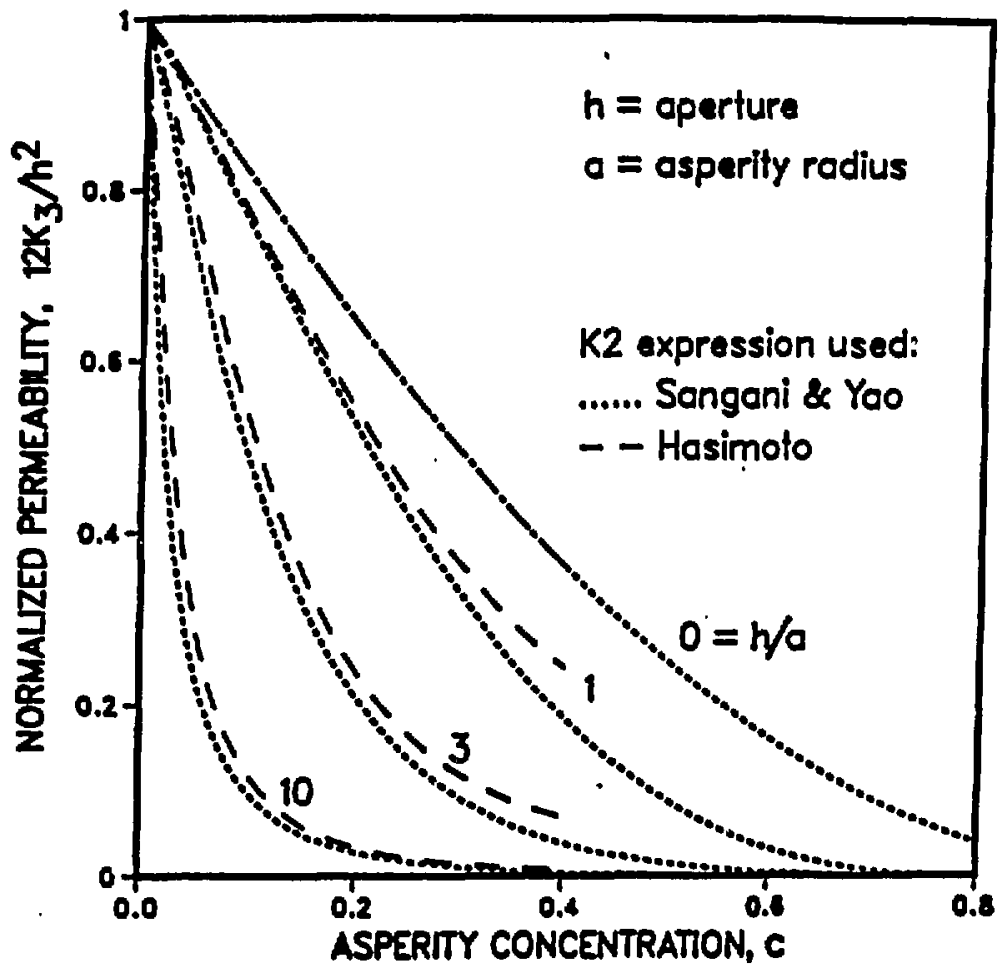

FIG. 5. Three-dimensional fracture permeability, using two different expressions for $\boldsymbol{K}_{\mathbf{2}}$. 


\section{DISCLAIMER}

This document was prepared as an eccount of wotk sponsored by the United Stukes Government. Neither the United Sinins Government nor any exency thereof, nor The Reents of the - Universily of California, nor any of their employees, makes any warranty, express cr implied, or assumes any legal liability or responsibility for the accuracy, completeness, of uxefulness of any information, apparatus, product, or process disclosed, or ' represents that its uxe would not infringe privately owned richts. 'Reference herein to any specific commercial products process, of service by its Irade name, trademark, manufacturer, or otherwise. does not necessarily constituke or imply its endorsement. recommendation, or favorine by the Unived Sules rovernment or any ayency thereof, or The Rezents of the University of Cali. - formin. The views and opinions of authors expressed herein do not necesurily state or reflect those of the United Sutes Government or any wency thereof or The Rewents of the University of California and shall nol be used for advertising or product endorsement purposes.

Luwrence Berkeley Laboratory is an equal opportunity employer. 